\title{
Temperature Dependence of the Absorption Coefficient in Doped Quantum Wells
}

\author{
M.R. Baldan ${ }^{\dagger}$, R.M. Serra ${ }^{\ddagger}$, P.D. Emmel ${ }^{\ddagger}$, and A. Ferreira da Silva* \\ INPE ${ }^{* \dagger}$ - LAS, 12.201-970 - São José dos Campos, SP, Brazil \\ UFSCar ${ }^{\ddagger}$ - Depart. de Física, 13565-905-São Carlos, SP, Brazil \\ USF ${ }^{\dagger}$ - Faculdade de Engenharia, 13251-900 - Itatiba, SP, Brazil
}

Received February 8, 1999

\begin{abstract}
In this work we present a calculation of the infrared optical absorption coefficient for a $\mathrm{Ga}_{1-x} \mathrm{Al}_{x} \mathrm{As}$ quantum well (QW) as a function of donor impurity concentration, compensation and temperature. We treat this problem using a Self-Consistent Method (SC) calculation taking into account donors as major impurities. The Variational Method was used to obtain the ground state wave-function of an electron bound to a donor impurity in a QW. The results show a shift of energy in the absorption coefficient.
\end{abstract}

\section{Introduction}

Many applications in opto-electronic devices are due to optical properties of quasi-two-dimensional systems. In particular the GaAlAs-GaAs quantum well is a popular system to study these properties. This kind of system is generated by several techniques by growing a layer of GaAs between two layers of GaAlAs.

The infrared absorption coefficient for a doped quantum well has been calculated by several authors $^{[1]-[10]}$. Recently Serra et al. ${ }^{[11]}$ calculated thermal properties of a lightly doped and compensated QW showing the Shottky anomaly in the specific heat. In this work we present the calculation of the optical absorption coefficient (OAC) for this kind of QW and we show its dependence on temperature and compensation. One important result is the presence of a threshold in the absorption energy.

\section{The Model}

We consider a lightly doped compensated QW. The compensation is defined by $k=N_{A} / N_{D}$, where $N_{A}$ and $N_{D}$ are the acceptor and donor densities respectively. Once there is a low concentration of dopants, donor impurities are far away from each other and we neglect the superposition of the electron wave-functions, so the unique kind of interaction present in our model is the electrostatic interaction between ionized impurities.
With this assumption the transitions are of intra-site type, in other words transitions between energy levels belong to the same impurity. At sufficiently low temperature all acceptors and an equal number of donors are ionized. The energy of the system depends on the distribution of neutral $\left(D^{0}\right)$ and ionized $\left(D^{+}\right)$donors and ionized acceptors $\left(A^{-}\right)$.

The electrostatic potential acts on the electron energy as a weak perturbation. As the temperature increases the tendency of electrons is to move from center to periphery of the QW, modifying the impurity configuration and increasing the energy of the system.[11]

The calculation was made using the self consistent method to determine the thermal properties of the system. From the initial charge density ${ }^{[10]}$ the electrostatic potential $\phi(z)$ is obtained via Poisson's equation. Once calculated the density of states, the chemical potential is obtained with the assumption of neutrality and the density of states is recalculated. The process continues until the convergence of the chemical potential is achieved.

The Hamiltonian of an electron bound to a shallow donor, placed inside a $\mathrm{QW}$ is given by ${ }^{[5]}$

$$
H\left(z_{k}\right)=-\frac{\hbar^{2}}{2 m^{*}} \nabla^{2}-\frac{e^{2}}{K u}+V(z)
$$

where $m^{*}$ is the effective mass of the electron, $K$ the effective dielectric constant of the QW, $e$ the electronic charge and $u=\sqrt{x^{2}+y^{2}+\left(z-z_{k}\right)^{2}}$ the distance be- 
tween the electron and the donor sited at $\left(0,0, z_{k}\right)$. $V(z)$ is the confining potential described, in the model of infinite barriers, as:

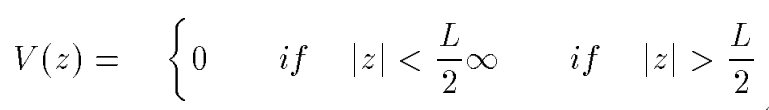

where $L$ is the width of the QW. The effective units used are:

$$
a^{*}=\frac{\hbar^{2} K}{m^{*} e^{2}}
$$

the effective Bohr radius (in GaAs $1 a^{*} \approx 100 \AA$ ) and

$$
R y^{*}=\frac{m^{*} e^{4}}{2 \hbar^{2} K^{2}}
$$

the effective rydberg (in GaAs $1 R y^{*} \approx 5.8 \mathrm{meV}$ ).

The $1 s$ and $2 p$ states are calculated using a standard variational method ${ }^{[9][5]}$. The variational wave functions are given by:

$$
\begin{gathered}
\Psi_{1 s}=A_{1 s} \cos \left(\frac{\pi z}{L}\right) e^{-K_{1 s} u} \\
\Psi_{2 p}=A_{2 p} \cos \left(\frac{\pi z}{L}\right) e^{i \phi} \rho e^{-K_{2 p} u}
\end{gathered}
$$

where $A_{1 s}, A_{2 p}$ and $K_{1 s}, K_{2 p}$ are the normalization constant and variational parameter to be determined. In Figure 1 we show the dependence of the energies of theses states on the impurity position.

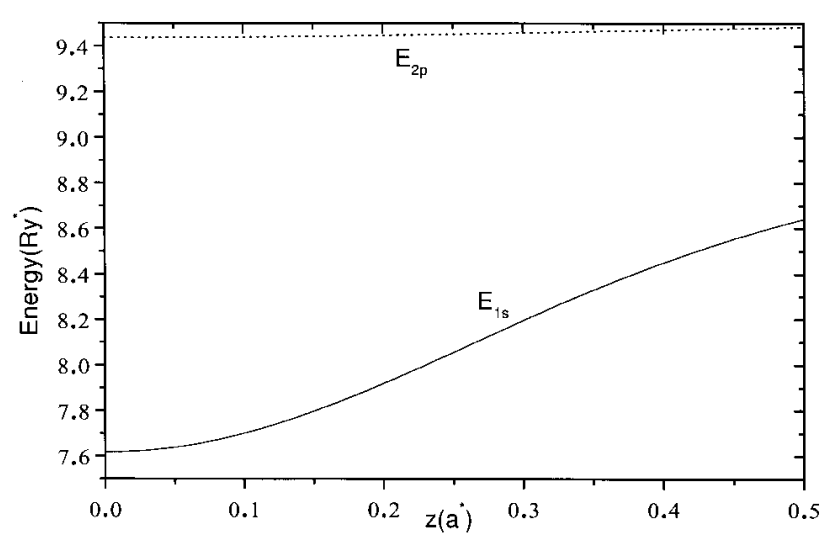

Figure 1. Energies of the states $1 \mathrm{~s}$ and $2 \mathrm{p}$.

The time average dissipation rate of electromagnetic energy is given by

$$
P=\sigma_{1}\left\langle E^{2}\right\rangle V
$$

where $\sigma_{1}$ is the real part of the material optical conductivity, the symbol $\langle\ldots\rangle$ is the time average of a physical quantity, $E$ is the radiation electric field, and $V$ is the volume of a single impurity.

From the Fermi golden rule we obtain the transition rate $(W)$ per unit of time between the impurity energy levels. Taking $\hbar \omega$ as the photon energy absorbed by a donor impurity we can write:

$$
P=W \hbar \omega
$$

so the absorption coefficient related to $k$-th neutral donor impurity, located at $z_{k}$ position is given by:

$$
\hbar \omega W\left(z_{k}, \omega\right)=\sigma_{1}\left(z_{k}, \omega\right)\left\langle E^{2}\right\rangle V
$$

In the long wave approximation we have:

$$
\sigma_{1}\left(z_{k}, \omega\right)=\frac{\pi e^{2} \omega}{V}\left|\mathbf{u} .\langle n|\mathbf{r}| m\rangle_{z_{k}}\right|^{2} \delta\left(E_{n m}\left(z_{k}\right)-\hbar \omega\right)
$$

where $\langle n|\mathbf{r}| m\rangle_{z_{k}}$ is the matrix element of the operator $\mathbf{r}$, taken between the states $n$ and $m$ of the $k$-th bound electron, and $\mathbf{u}$ is the polarization of the absorbed photon with frequency $\omega$, and wave vector $\mathbf{k}$,perpendicular to the barriers.

The absorption coefficiente for the system is ob- tained summing up the contributions of all neutral donors. Taking an uniform profile of donor impurities, making use of Fermi-Dirac distribution function $\left(f_{F D}\left(E_{1 s}, T\right)\right)$ and considering transitions between $1 s$ and $2 s$ levels we have: 


$$
\sigma_{1}(\omega, T)=N_{D} \pi e^{2} \omega f_{F D}\left(E_{1 s}\left(z_{0}\right), T\right)\left|\mathbf{u} .\langle 1 s|\mathbf{r}| 2 p\rangle_{z_{0}}\right|^{2} D_{J}(\omega)
$$

where $z_{0}$ is the donor position in which the transition takes place, $D_{J}$ is the joint density of states and $N_{D}=1 / V$

\section{Results}

We have performed the calculations with $L=100 A^{\circ}$, $N_{D}=10^{9}$ donor $/ \mathrm{cm}^{2}$ for different compensations and temperatures. Figure 2 shows the difference in energy between $1 \mathrm{~s}$ and $2 \mathrm{p}$ states. In the limit of low temperatures almost all neutral donors will be located at the middle of the QW so that only high energy photons will be absorbed. As temperature increases electrons flow from the center of the QW to its periphery in order to increase the energy of the system. As a consequence there will be an increase in the number of the neutral donors at the periphery of the QW so that the threshold energy $\left(E_{t h}\right)$, the minimal energy for the occurrence of the transition, turns down. In this work we consider the threshold energy as the intersection of the tangent line at the inflection point of the curve $\sigma_{1} \times E$ with the energy axis.

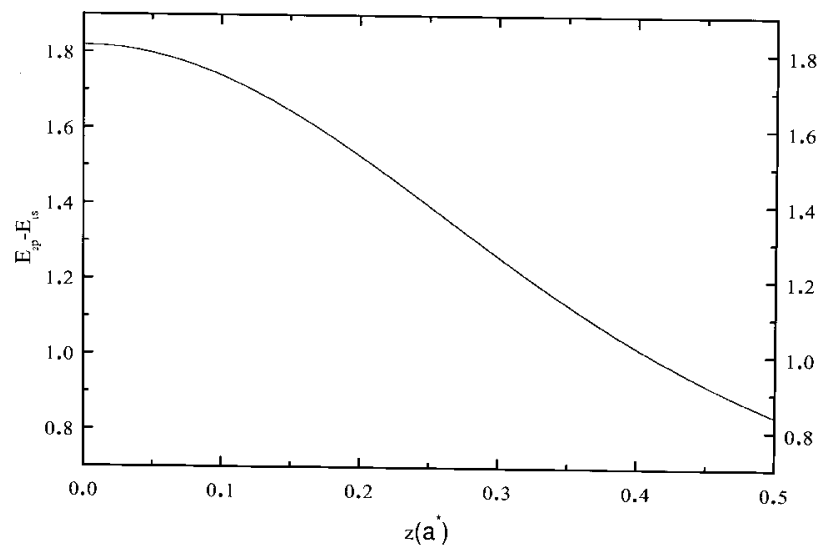

Figure 2. Energy difference between $E_{2 p}-E_{1 s}$ of the an electron bound to a donor located at $z$ postition in a $\mathrm{QW}$.

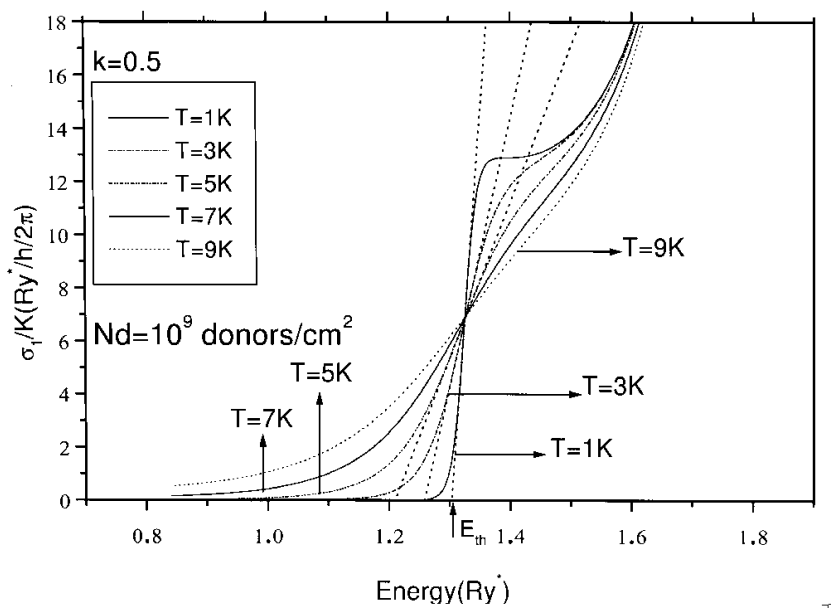

Figure 3. The effect of the temperature on the absorption coefficient for $\mathrm{k}=0.5$.

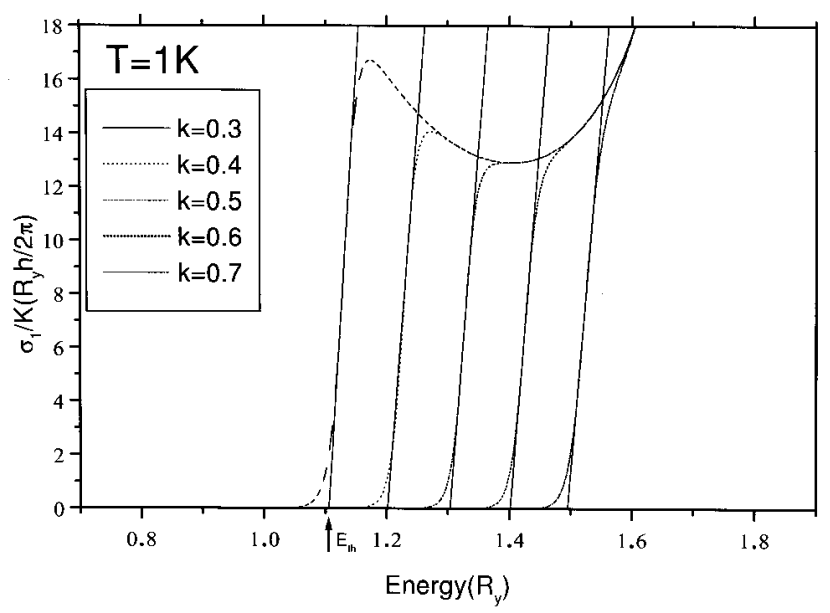

Figure 4. The effect of the compensation on the absorption coefficient, with $\mathrm{L}=100 \mathrm{~A}^{\circ}$ and temperature of $\mathrm{T}=1 \mathrm{~K}$.

Figure 3 shows the absorption coefficient for a compensation of 0.5 and the temperature varying from $1 \mathrm{~K}$ to $9 \mathrm{~K}$. If we keep the temperature constant changing the compensation, we notice also a change in the threshold energy as we see in figure 4 . This effect can be explained by the fact that as the compensation increases, the number of neutral donors decreases so that when $k=1$ all donors will be ionized and there will not be absorption. At low temperatures the configuration of neutral donors is almost equal to that one of the ground state with excited donors located at the periphery of the 
QW so to minimize the energy of the system. As the compensation decreases the number of neutral donors increases, the regions far from the center of the QW become populated by neutral donors and the threshold energy is shifted down. The shape, in figure 4, is very similar to that one calculated by Emmel et al at $T=O K[6]$

Another important effect is the enhancement of the absorption coefficient due to the increasing of the donor density. Equation 11 shows that the coefficient of absorption is proportional to $N_{D}$. Our treatment is limited to low donor density since we consider isolate impurities. Although experimental results for the dependence of absorption coefficient on temperature and compensation are not yet available we believe that the absorption threshold energy could be further used to diagnose this kind of doped QW.

\section{References}

[1] E. Glaser, B.V. Shanabrook, R.L. Hawkins and
W. Beard, Phya. Rev. B 36, 8185 (1987).

[2] E. Glaser, W.J. Moore, B.V. Shanabrook, R.L. Hawkins, R.J. Wagner and D. Musser, J. of Phys. 48, 239 (1987).

[3] B.V.J. Shanabrook, Physica B 146, 121 (1987).

[4] L.E. de Oliveira and G.D. Mahan, Phys. Rev. B 47, 2406 (1993)

[5] P.D. Emmel and I.C. da Cunha Lima, Solid State Commun. 89, 725 (1994).

[6] P.D. Emmel and I.C. da Cunha Lima, Solid State Commun. 79, 431 (1991).

[7] G.N. Carneiro, G. Weber and L.E. de Oliveira, Semicond. Sci. Technol. 10, 41 (1995).

[8] A. Ferreira da Silva, I.C. da Cunha Lima, P.D. Emmel, Superlattices and Micros. 16, 335 (1994).

[9] G. Bastard, Phys. Rev. B 24, 4714 (1981).

[10] B.C.F. Colchesqui, P.D. Emmel, E.A. de Andrada e Silva and I.C. da Cunha Lima. Phy. Rev. B 40, 18, 12513 (1989).

[11] R.M. Serra and P. Daniel Emmel, Brazilian J. of Physics. 27/A, 295 (1997). 